\title{
MR Image Denoising Algorithm Based on Variance Stable Transformation and LSM-NLR
}

\author{
Liling $\mathrm{Yu}^{+}$, Binfeng Xu, Gangping Zhang, Wenping Liu, Haijun Chen, Jingyu Guo \\ Guangdong Food and Drug Vocational College, Guangzhou, 510520, China
}

\begin{abstract}
The denoising algorithm based on Laplacian scale mixing model and non-local low-rank approximation (LSM-NLR) has a very good denoising effect for mixed gaussian white noise images, but the suppression effect is not ideal for spatial variation Rician noise in MR images with low signal-to-noise ratio. Therefore, this paper proposes a new denoising method combining variance stable transformation and LSMNLR. This method has used the noise estimated by modified median absolute deviation to carry out the variance stable transformation of the noise image amplitude, so that the noise is independent of the signal amplitude and spatial position. LSM-NLR algorithm can be used to suppress the noise of transformed image, and finally obtain the unbiased denoised image through the variance stable inverse transformation. Experimental results have demonstrated that the proposed algorithm can suppress MR image noise effectively and protect details of image. The value of PSNR and SSIM were slightly higher in the simulation experiment. The NIQE value and BIQI value of the proposed algorithm were slightly lower and higher from the de-noising results of Real breast MR images.
\end{abstract}

Keywords: Rician noise, variance-stabilization transformation, LSM, Nonlocal Low-rank.

\section{Introduction}

Magnetic resonance image (MRI) has unique advantages in disease diagnosis and has become one of the important means of disease examination. However, MR images are often disturbed by Rician noise during the acquisition process. The presence of noise will reduce the quality of the image, bring difficulties to medical diagnosis, and is not conducive to subsequent image analysis and processing. Therefore, it is necessary to remove the Rican noise from the MR image.

At present, the denoising algorithms based on the improved BM3D algorithm [1] and based on non-local filtering have been widely used by researchers to suppress Rician noise. Fio [2] has proposed the use of variance-stabilization transformations (VST) to transform MR images, combined with the BM3D or BM4D algorithm to remove Rician noise effectively. Manjón proposed an adaptively adjusted non-local mean algorithm [3] for suppressing the Rician noise in MR images and achieved good denoising effects. However, the algorithm based on non-local mean is complex, with many parameters, and it is not easy to find the optimal parameters, and the calculation is time-consuming. Ai [4] proposed a denoising algorithm combining dual-domain filtering and guided filtering, which can achieved good denoising effect, but did not have the ability to suppress the spatial variation of noise.

Huang Tao [5] has proposed a denoising method based on Laplacian Scale Mixture Modeling and Nonlocal Low-rank (LSM-NLR) to remove the mixed Gaussian white noise and impulse noise, which pointed out that the denoising effect of the LSM-NLR algorithm is equivalent to that of the BM3D algorithm for removing the Gaussian white noise. The Rician noise in the MR image obeys the Rayleigh distribution at low signal-to-noise ratio, so there is no advantage in removing Rician noise by using the LSM-NLR algorithm. The variance stable transformation is used to transform the spatially varying Rician noise into a

+ Corresponding author. Tel.: + 020-29164611; fax: +020-29164611.

E-mail address: lilingyu124@126.com. 
constant level of additive noise in the MR image, and then the general denoising algorithm is used to suppress the noise. The accurate estimation of the noise level has a great impact on the image effect after VST. Therefore, this paper has proposed a denoising method based on VST and LSM-NLR algorithm to remove the spatially varying Rician noise in the MR image.

\section{New denoising method based on VST and LSM-NLR algorithm}

\subsection{Variance Stable Transformation Algorithm}

During MR imaging, the original K-space data is disturbed by complex Gaussian noise. The amplitude of the MR image is the square root of two independent Gaussian random variables. VST algorithm [6] is a mathematical transformation method that can convert heteroscedastic data to homoskedasticity, removing the dependence of Rician noise variance and noiseless image amplitude. In this paper, the modified median absolute deviation (MAD) is used to estimate the Rician noise level in the MR image. The estimated noise level is used to stabilize the variance of each pixel in the MR image, so that the noise level of the entire image is approximately constant everywhere. The formula is as follows:

$$
M(i, j) \leftarrow M(i, j) / \sigma(i, j)
$$

Where $M$ is the MR image amplitude, $\sigma$ is the standard deviation of Gaussian noise in real and virtual images, $i$ and $j$ are the pixel. Equation (1) is actually a homomorphic process on the MR image, so that the Rician noise of the entire image is approximately converted into a constant level of additive Gaussian noise.

\subsection{LSM-NLR Noise Removal Model}

In theory, after the variance stable transformation, the Rician noise is approximately converted into a constant level of additive Gaussian noise, but the accurate estimation of the noise level has a great influence on the result of the variance stable transformation of the MR image containing the Rician noise. This paper assumes that the transformed image $y$ after the variance stable transformation has a singular point, so $y$ can be expressed as follows:

$$
y=x+w+s
$$

Where $x$ is the denoised image, $w$ is a constant level of Gaussian white noise, and $s$ is a singular point.

Because the noise level of the stable variance transformation is approximately constant Gaussian white noise, the variance is expressed by $\sigma_{\omega}$. From equation (2), we know that the problem of denoising will be transformed into a problem of solving $x$ and $s$, and find the appropriate $x$ and $s$ so that their conditional posterior probability is the largest. The formula is as follows:

$$
\{x, s\}=\arg \max P\left(x, s, \sigma_{w} \mid y\right)=\arg \max \log P(y \mid x, s)+\log P(x)+\log P(s)
$$

Suppose $P(s)$ follows the Laplace distribution, Equation (3) căn be transformed into an energy minimization problem.

$$
(x, s)=\underset{x, s}{\arg \min } \frac{1}{2 \sigma_{w}^{2}}\|y-x-s\|_{2}^{2}+\lambda\|s\|_{1}^{1}+P(x)
$$

Where $\lambda=1 / \theta, \theta$ is the standard deviation of $s_{i}$, and $P(x)$ is the prior term of the image after denoising.

Using the Laplacian scale hybrid model, the singular point $s$ is decomposed into a point product of the recessive variable factor $\gamma$ and the Laplacian variable $\eta$. The energy minimization problem becomes the following formula:

$$
(x, \gamma, \eta)=\underset{x, \gamma, \eta}{\arg \min }\|y-x-\gamma \cdot \eta\|_{2}^{2}+4 \sigma_{w}^{2} \sum_{i} \log (\gamma+\varepsilon)+2 \sqrt{2} \sum_{i}|\eta|+P(x)
$$

Where $\varepsilon=10^{-5}$ is used to ensure the stability of the calculation.

The selection of $P(x)$ priors is an important step for image denoising. This paper has made a non-local low-rank model to model the priors. The non-local low-rank model uses a block matching method based on Euclidean distance to find $k$ similar image blocks similar to the sample block in the search range, and expands each similar image block into columns to aggregate into a matrix $Z_{i}$ ( $i$ is a pixel point coordinates), $Z_{i}$ has a low rank attribute. Assuming $Z_{i} X=\left[R_{i, 0} X R_{i, 1} X \ldots R_{i, k-1} X\right]$, which represents a matrix of similar image blocks similar to the sample block $x_{i}$, the non-local low-rank image prior can be expressed as: 


$$
P(x) \propto \prod \exp \left(-\zeta \operatorname{rank}\left(Z_{i} x\right)\right)
$$

Where $\zeta$ is the regularization parameter. Substituting eiquation (6) into equation (4), the problem of energy minimization can be expressed as:

$$
(x, \gamma, \eta)=\arg \min \|y-x-\gamma \cdot \eta\|_{2}^{2}+4 \sigma_{w}^{2} \sum \log (\gamma+\varepsilon)+2 \sqrt{2} \sum|\eta|+\zeta \sum \operatorname{rank}\left(Z_{i} x\right)
$$

Dong [7] has proposed to use smoothth non-convex function ${ }^{i} L(X, \varepsilon)$ to approximate ránk $(\bullet)$, and pointed out that logdet $(\bullet)$ has a better low rank approximation [8], $L(X, \varepsilon)$ is defined as:

$$
L(X, \varepsilon)=\sum_{r=1}^{r_{0}} \log \left(\sigma_{r}(X)+\varepsilon\right)
$$

Among them, $X$ is a low-rank matrix, $\sigma_{r}(X)$ denotes the $r^{\text {th }}$ singular value of $X, r_{0}=\min (n, m)$, and $m$ and $n$ are image sizes. Therefore, equation (7) can be expressed as:

\subsection{Iterative Optimization}

$$
(x, \gamma, \eta)=\underset{x, \gamma, \eta}{\arg \min }\|y-x-\gamma \cdot \eta\|_{2}^{2}+4 \sigma_{w}^{2} \sum_{i} \log (\gamma+\varepsilon)+2 \sqrt{2} \sum_{i}|\eta|+\zeta \sum_{i} L\left(Z_{i} x, \varepsilon\right)
$$

For the solution of equation (9) in this paper, we use an alternating iterative optimization method to solve the hidden variable factor $\gamma$, Laplace variable $\eta$, and the denoised image $x$. Fixing $\mathrm{x}$ and $\eta$, the calculation formula of recessive variable factor $\gamma$ is as follows:

$$
\gamma=\underset{\gamma}{\arg \min }\|y-x-\gamma \cdot \eta\|_{2}^{2}+4 \sigma_{w}^{2} \sum_{i} \log (\gamma+\varepsilon)
$$

Fixing $x$ and $\gamma$, the Laplace variable $\eta$ is calculated as follows:

$$
\eta=\underset{\eta}{\arg \min }\|y-x-\gamma \cdot \eta\|_{2}^{2}+2 \sqrt{2} \sum_{i}\left|\eta_{i}\right|
$$

Calculate the solution hidden variable factor $\gamma$ and Laplacian variable $\eta$, then the formula for denoised image $x$ is as follows:

$$
x=\arg \min \|y-x-\gamma \cdot \eta\|_{2}^{2}+\zeta \sum_{i} L\left(Z_{i} x, \varepsilon\right)
$$

Since the LSM-NLR denoising process is performed in the transform domain, the last step needs to perform inverse variance stable transformation to finally obtain an unbiased denoised image.

\section{Result/Experiment}

\subsection{Simulated MR Image Experiments}

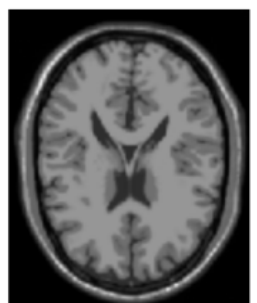

(a)

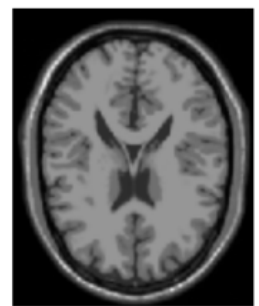

(a)

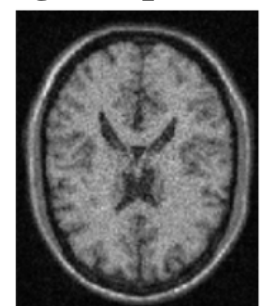

(b)

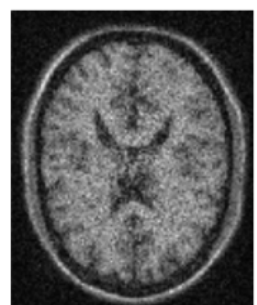

(b)

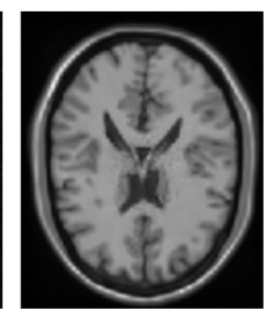

(c)

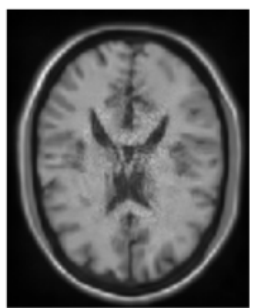

(c)

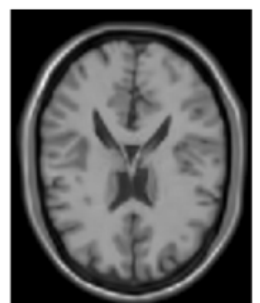

(d)

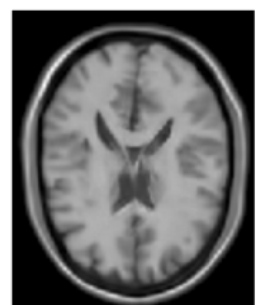

(d)

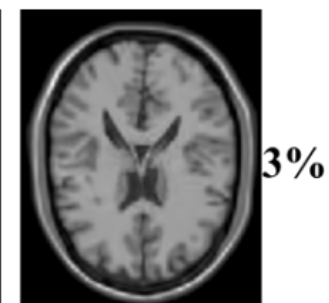

(e)

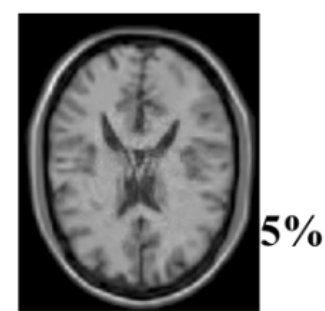

(e)

Fig. 1: The denoising results of different methods: (a) Original image; (b) Noisy image;(c) Denoised result of the LSMNLR;(d) Denoised result of the proposed method;(e) Denoised result of VST-BM3D

The de-noising effect of the simulation experiments in this paper is evaluated using three aspects: image visual effects, Peak Signal to Noise Ratio (PSNR), and Structural Similarity Index Metric (SSIM) [9]. Fig.1 has showed the denoising results obtained by using different methods at different noise levels for T1 
weighted images when the simulated noise levels are 3\% and 5\% respectively. As can be seen from Fig.1, this method can better suppress the spatial variation of noise. This method can better maintain the details and edge information of the original image. The noise of the LSM-NLR method in the area of low signal-to-noise ratio is not effectively suppressed. Especially the larger the noise level, the worse the ability of the LSMNLR method to suppress the Rician noise.

The T1 weighted image with noise is denoised using three different methods. The three denoising methods have different PSNR and SSIM values after denoising at different noise levels, as shown in Table 1. As can be seen from the table, the values of PSNR and SSIM of the method in this paper are slightly higher. However, as the noise level increases, the denoising effect of each denoising algorithm decreases significantly.

Table 1: Comparison of MR image denoising performance using different methods

\begin{tabular}{|c|c|c|c|c|c|c|}
\hline $\begin{array}{c}\text { noise levels } \\
1 \%\end{array}$ & \multicolumn{2}{|c|}{ LSM-NLR } & \multicolumn{2}{l|}{ VST-BM3D } & \multicolumn{2}{c|}{ VST-LSM-NLR } \\
\cline { 2 - 7 } & PSNR/dB & SSIM/dB & PSNR/dB & SSIM/dB & PSNR/dB & SSIM/dB \\
\hline $1 \%$ & 37.3626 & 0.8824 & 38.8532 & 0.9824 & 39.0582 & 0.9853 \\
\hline $2 \%$ & 31.9623 & 0.8126 & 33.5730 & 0.9469 & 34.4348 & 0.9622 \\
\hline $3 \%$ & 29.1313 & 0.7751 & 31.6012 & 0.9149 & 32.7263 & 0.9431 \\
\hline $4 \%$ & 26.7693 & 0.7413 & 30.1522 & 0.8911 & 30.5147 & 0.9115 \\
\hline $5 \%$ & 24.7260 & 0.6914 & 28.6117 & 0.8543 & 29.2690 & 0.8879 \\
\hline $6 \%$ & 23.0939 & 0.6568 & 27.2837 & 0.8232 & 27.7686 & 0.8550 \\
\hline $7 \%$ & 20.9879 & 0.5419 & 26.0039 & 0.7824 & 26.7543 & 0.8236 \\
\hline $8 \%$ & 18.9761 & 0.4844 & 25.0130 & 0.7622 & 25.4949 & 0.7935 \\
\hline
\end{tabular}

\subsection{Real MR Image Experiments}

This paper has used real breast MR images as the research object. Fig.2 shows the denoising effect of a real MR image using different methods. Because the noise level of the real MR image is relatively small, the three denoising methods can suppress the noise. From the visual point of view of Fig.2, the denoised images by LSM-NLR are slightly excessively smooth, however the organizational structure and details are clear by the proposed method.

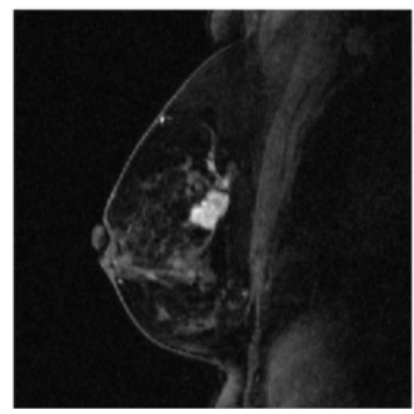

(a)

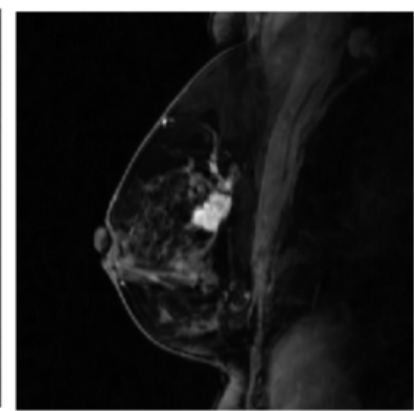

(b)

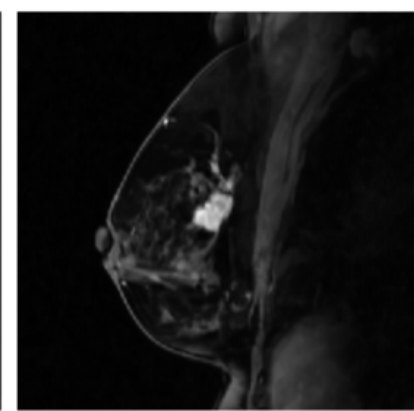

(c)

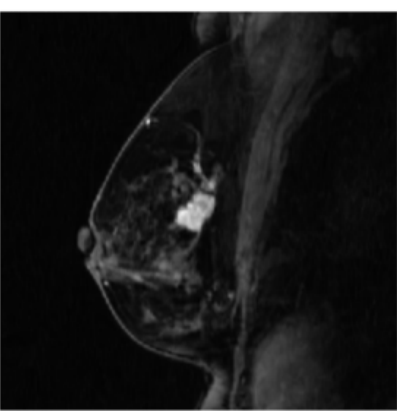

(d)

Fig. 2: The experimental results of real MR image. (a) Noisy image; (b) Result of LSM-NLR; (c) Result of our proposed method; (d) Result of VST-BM3D

Since the real noise image does not have a corresponding reference image, in order to evaluate the denoising effect, the Natural Image Quality Evaluator (NIQE) [10] and Blind Image Quality Index (BIQI) are used to evaluate the effect of denoised image. When the reference image is not available, the NIQE metric can better evaluate the image quality. The image quality is better when the value of NIQE is smaller. The BIQI index is tested using the Laboratory for Image \& Video Engineering database. The closer the BIQI value is to 0 , the worse the image quality, and the closer to 1 , the better the image quality. Table 2 lists the NIQE and BIQI of eight noisy images and the denoised images using three different methods. The NIQE value corresponding to the proposed algorithm is lower, and the BIQI value is slightly higher. 
Table 2: Comparison of real MR image denoising performance

\begin{tabular}{|c|c|c|c|c|c|c|c|c|}
\hline \multirow{2}{*}{ label } & \multicolumn{2}{|c|}{ Noise image } & \multicolumn{2}{c|}{ LSM-NLR } & \multicolumn{2}{l|}{ VST-BM3D } & \multicolumn{2}{l|}{ VST-LSM-NLR } \\
\cline { 2 - 9 } & NIQE & BIQI & NIQE & BIQI & NIQE & BIQI & NIQE & BIQI \\
\hline 1 & 9.4759 & 0.5616 & 5.9812 & 0.7249 & 6.0306 & 0.9214 & 5.4821 & 0.8967 \\
\hline 2 & 9.3351 & 0.5684 & 5.7409 & 0.8824 & 5.4917 & 0.8558 & 5.3022 & 0.8967 \\
\hline 3 & 9.8894 & 0.5088 & 5.8542 & 0.8688 & 6.6238 & 0.8525 & 5.7287 & 0.8771 \\
\hline 4 & 12.1505 & 0.5518 & 6.2129 & 0.8625 & 7.1252 & 0.8234 & 6.0949 & 0.8778 \\
\hline 5 & 9.9368 & 0.5478 & 5.5991 & 0.8037 & 5.7503 & 0.8383 & 5.1447 & 0.8104 \\
\hline 6 & 12.8242 & 0.4935 & 8.0722 & 0.8805 & 7.1407 & 0.8961 & 5.7924 & 0.9326 \\
\hline 7 & 11.8662 & 0.5704 & 7.0102 & 0.7854 & 7.2742 & 0.8078 & 5.6159 & 0.8347 \\
\hline 8 & 8.5510 & 0.5248 & 5.1857 & 0.8718 & 5.8536 & 0.8055 & 5.1345 & 0.8777 \\
\hline
\end{tabular}

\section{Conclusion}

Aiming at the Rican noise with spatial variation of noise level in MR image, MAD is used to estimate the noise level to perform variance stable transformation on the amplitudes of the image, so that the noise is independent of the signal amplitude and spatial position, which has made a big impact effect. This paper has used the estimated noise to stabilize the variance of the MR image so that the Rician noise is converted into additive Gaussian white noise. Then the LSM-NLR algorithm was employed to suppress the noise of the transformed image. The simulation experiment results show that the PSNR and SSIM corresponding to the proposed algorithm are relatively high, and the details and structure of the MR image are well maintained. Since the Rician noise of real MR image is relatively small, three denoising methods visually can all suppress the spatial variation of noise. The proposed denoising method can achieve a slightly lower NIQE value and a higher BIQI value.

\section{Acknowledgements}

The authors wish to acknowledge project funding support from Guangdong Province Medical Scientific Research Foundation No.B2017093, and the Research Gant of Guangdong Food and Drug Vocational College No.2018ZR032. Guangdong Province Colleges and Universities Young Innovative Talents Project NO.2019GKQNCX062.The authors declare no competing financial interests.

\section{References}

[1] Dabov K, Foi, A, Katkovnik, V, et al. Image Denoising by Sparse 3-D Transform-Domain Collaborative Filtering. IEEE Transactions on Image Processing. 2007.16(8):2080-2095.

[2] M. Maggioni, V. Katkovnik, K. Egiazarian, and A. Foi, "A Nonlocal Transform-Domain Filter for Volumetric Data Denoising and Reconstruction”. IEEE Transactions on Image Processing. 2013. 22 (1): 119-133.

[3] José V. Manjón, Pierrick Coupé, Luis Martí-Bonmatí, et al. Adaptive non-local means denoising of MR images with spatially varying noise levels. Journal of Magnetic Resonance Imaging Jmri. 2010. 31(1):192-203.

[4] Ai Ling-mei, Ren Yang-hong. Fast denoising method for medical MR image based on dual-domain filtering and guided image filtering. Optoelectronics · Laser. 2018. 277(7):101-110.

[5] Huang T, Dong W, Xie X, et al. Mixed Noise Removal via Laplacian Scale Mixture Modeling and Nonlocal Lowrank Approximation. IEEE Transactions on Image Processing. 2017.pp.1-1.

[6] Foi A. Noise estimation and removal in MR imaging: The variance-stabilization approach. IEEE International Symposium on Biomedical Imaging: from Nano to Macro. 2011.

[7] Dong Weisheng, Shi Guangming, Li Xin, et al. Compressive sensing via nonlocal low-rank regularization. IEEE Transactions on Image Processing. 2014. 23(8):3618-3632.

[8] Zhao Hui, Zhang Jing, Zhang Le, et al. Compressed Sensing Image Restoration Based on Non-local Low Rank and Weighted Total Variation. Journal of Electronics \& Information Technology.2019.41:1-8. 
[9] Xiao Jia, Zhang Junhua, Mei Liye. Salt and pepper noise denoising based on improved BM3D algorithm. Computer Engineering and Applications. 2018. 54(21):175-180+212.

[10] Mittal A, Fellow, IEEE, et al. Making a 'Completely Blind' Image Quality Analyzer. IEEE Signal Processing Letters.2013. 20(3):209-212. 